\title{
Oral S-1 with 24-h Infusion of Irinotecan plus Bevacizumab versus FOLFIRI plus Bevacizumab as First-Line Chemotherapy for Metastatic Colorectal Cancer: An Open-Label Randomized Phase II Trial
}

\author{
Sotaro Sadahiro Toshiyuki Suzuki Kazutake Okada Gota Saito \\ Hiroshi Miyakita Takashi Ogimi Lin Fung Chan Yutaro Kamei \\ Department of Surgery, Tokai University, School of Medicine, Isehara, Japan
}

\author{
Keywords \\ Colorectal cancer · S-1 · Irinotecan · Bevacizumab · 24-h \\ infusion · Phase II study · FOLFIRI
}

\begin{abstract}
Background: FOLFIRI plus bevacizumab have been widely used as first-line treatment for metastatic colorectal cancer ( $\mathrm{mCRC}$ ). Pharmacokinetics and pharmacodynamics suggested a low dose of irinotecan given as a long-term infusion is expected to enhance antitumor activity. We conducted a randomized phase II study to compare oral S-1 with a 24-h infusion of irinotecan plus bevacizumab versus FOLFIRI plus bevacizumab. Methods: The subjects comprised 120 chemotherapy-naïve patients with $\mathrm{mCRC}$. The study group received a 24-h infusion of irinotecan at a dose of $125 \mathrm{mg} / \mathrm{m}^{2}$ on days 1 and 15 , combined with oral $\mathrm{S}-180 \mathrm{mg} / \mathrm{m}^{2}$ on days 1-14 (24h-SIRI/B). The FOLFIRI/B group received irinotecan at a dose of $150 \mathrm{mg} / \mathrm{m}^{2}, 5$-fluorouracil given at a dose of 400 $\mathrm{mg} / \mathrm{m}^{2}$ as a bolus injection and at a dose of $2,400 \mathrm{mg} / \mathrm{m}^{2}$ as a 46-h infusion, and $200 \mathrm{mg} / \mathrm{m}^{2}$ leucovorin on days 1 and 15 . Bevacizumab was given at a dose of $5.0 \mathrm{mg} / \mathrm{kg}$ on days 1 and 15 in both groups. Treatment was repeated every 4 weeks. The primary endpoint was 1-year progression-free survival
\end{abstract}

\begin{tabular}{ll}
\hline karger@karger.com & (C) 2020 The Author(s) \\
www.karger.com/ocl & Published by S. Karger AG, Basel Karger \\
& This article is licensed under the Creative Commons Attribution- \\
Karger & NonCommercial-NoDerivatives 4.0 International License (CC BY- \\
NC-ND) (http://www.karger.com/Services/OpenAccessLicense). \\
Usage and distribution for commercial purposes as well as any dis- \\
tribution of modified material requires written permission.
\end{tabular}

(PFS). Secondary endpoints were PFS, response rates (RR), overall survival (OS), and adverse events (AEs). Results: From December 2013 through January 2018, 120 patients were randomly assigned, 61 patients to the $24 \mathrm{~h}-\mathrm{SIRI} / \mathrm{B}$ and $59 \mathrm{pa}-$ tients to the FOLFIRI/B. The median follow-up period was 22.8 months. The 1 -year PFS rate was $43.14 \%$ in the $24 \mathrm{~h}$ $\mathrm{SIRI} / \mathrm{B}$ arm and $19.15 \%$ in the FOLFIRI/B arm (HR $=0.312$ [95\% Cl 0.13-0.78], $p=0.01)$. The median PFS was 10.2 months ( $95 \% \mathrm{Cl} 8.8-14.3)$ and 10.0 months $(95 \% \mathrm{Cl} 7.4-11.0)$, and the median OS was 29.7 months $(95 \% \mathrm{Cl} 22.9-43.9)$ and 28.8 months $(95 \% \mathrm{Cl} 18.4-\mathrm{ND})$, respectively $(p=0.3758, p=$ 0.8234 ). The overall RR was 86.3 and $61.7 \%$, respectively ( $p=$ $0.0053)$. AEs were similar. Conclusions: Our results show that the $24 \mathrm{~h}-\mathrm{SIRI} / \mathrm{B}$ regimen is an effective and reasonably welltolerated regimen for the first-line treatment of $\mathrm{mCRC}$.

(c) 2020 The Author(s)

Published by S. Karger AG, Basel

\section{Introduction}

Fluorouracil (FU)-based doublets, including oxaliplatin or irinotecan, plus bevacizumab have been widely used as first-line treatment for metastatic colorectal cancer (mCRC) [1-3]. Combination regimens of FU and ox- 
aliplatin, such as FOLFOX, CapeOx, and SOX (S-1 plus oxaliplatin), are more commonly used in clinical practice than combination regimens of FU and irinotecan, such as FOLFIRI. However, peripheral neuropathy associated with oxaliplatin often leads to early treatment withdrawal and negatively affects treatment continuity. Prolonged peripheral neuropathy affects patients' daily life activities and their quality of life $[4,5]$. In contrast, the combination regimens of FU and irinotecan can be continued for long periods in many patients by managing early major toxicity such as diarrhea.

Irinotecan is usually given as a 90 -min intravenous infusion biweekly or triweekly. However, the cytotoxic effects of irinotecan are specific to the S-phase of the cell cycle, and low doses of irinotecan given by protracted infusion or frequent bolus injection can prevent the saturation of carboxylesterases, enzymes that convert irinotecan to its active metabolite SN-38, and thereby enhance antitumor activity $[6,7]$. We have previously performed several clinical trials of a 24 -h continuous intravenous infusion of irinotecan, given biweekly, combined with oral FUs (UFT and UFT plus leucovorin), in patients with $\mathrm{mCRC}$ and reported that these regimens are therapeutically useful and have low incidences of diarrhea and other adverse events $[8,9]$. To date, however, no randomized clinical trial has compared a $24-\mathrm{h}$ continuous infusion of irinotecan with a standard 90 -min infusion of irinotecan.

S-1 is an oral anticancer drug that combines tegafur, a prodrug of 5-FU, with gimeracil and potassium oxonate in a molar ratio of 1:0.4:1 $[10,11]$. We previously performed a combination chemotherapy with oral S-1 and biweekly 24-h infusions of irinotecan plus bevacizumab in patients with $\mathrm{mCRC}$ and showed that this regimen appears to be highly active and well tolerated both as firstline and second-line chemotherapy for $\operatorname{mCRC}[12,13]$.

In this open-label randomized phase II trial, we compared combination treatment consisting of oral S-1 and a 24-h infusion of irinotecan plus bevacizumab with conventional FOLFIRI plus bevacizumab as first-line chemotherapy for mCRC.

\section{Patients and Methods}

\section{Patients}

The main inclusion criteria were as follows: histologically confirmed colorectal cancer (CRC), unresectable or recurrent CRC with measurable or evaluable disease, UGT1A1*28 genotype $(6 / 6$ or 6/7), an age of 20 years or older, an Eastern Cooperative Oncology Group performance status (ECOG PS) of 0 or 1, a life expectancy of at least 3 months, adequate organ function, and no previous chemotherapy or prior adjuvant chemotherapy for CRC with- in 3 months before enrollment. The main exclusion criteria were as follows: active infection, bowel obstruction, interstitial pneumonia and pulmonary fibrosis, uncontrolled diabetes, pleural effusion or ascites, pregnancy and lactation, and known drug allergies. Patients with metastasis or recurrence only in the liver were excluded because they were enrolled in clinical trials of other regimens, including hepatic artery infusion.

\section{Trial Design and Treatment}

This trial was a two-group, open-label, randomized, phase II trial conducted at Tokai University Hospital. Patients were randomly assigned in a 1:1 ratio to receive oral S-1 with a 24 -h infusion of irinotecan plus bevacizumab (24h-SIRI/B) or FOLFIRI plus bevacizumab (FOLFIRI/B). Randomization was carried out centrally, with minimization stratified by the number of metastatic sites $(1 / \geq 2)$, the primary lesion (colon/rectum), the primary site (absent/present), and ECOG PS (0/1).

The $24 \mathrm{~h}-\mathrm{SIRI} / \mathrm{B}$ regimen comprised irinotecan $\left(125 \mathrm{mg} / \mathrm{m}^{2}\right)$, given as a 24-h intravenous infusion on days 1 and 15, S-1 (80 mg/ $\mathrm{m}^{2}$ ), given orally in two divided daily doses on days $1-14$, and bevacizumab $(5.0 \mathrm{mg} / \mathrm{kg})$, given as an intravenous infusion on days 1 and 15 of each 4 -week cycle. The FOLFIRI/B regimen comprised irinotecan $\left(150 \mathrm{mg} / \mathrm{m}^{2}\right)$, given as a 90 -min intravenous infusion, and l-leucovorin $\left(200 \mathrm{mg} / \mathrm{m}^{2}\right)$, given as a 120 - $\mathrm{min}$ intravenous infusion, an intravenous bolus 5 -FU $\left(400 \mathrm{mg} / \mathrm{m}^{2}\right)$, a $46-\mathrm{h}$ continuous intravenous infusion of 5 -FU $\left(2,400 \mathrm{mg} / \mathrm{m}^{2}\right)$, and bevacizumab $(5.0 \mathrm{mg} / \mathrm{kg})$, given as an intravenous infusion on days 1 of each 2 -week cycle.

Immediately before the infusion of irinotecan, the patients were confirmed to have a leukocyte count of $\geq 3,000 / \mu \mathrm{L}$, a neutrophil count of $\geq 1,500 / \mu \mathrm{L}$, and a platelet count of $\geq 100,000 / \mu \mathrm{L}$, and to be free of diarrhea, infection, or fever suggestive of infection $\left(\geq 38^{\circ} \mathrm{C}\right)$, grade 1 or lower levels of serum total bilirubin, serum aspartate aminotransferase, and alanine aminotransferase, and grade 2 or lower other non-hematologic toxicities.

Bevacizumab was given to patients who had grade 2 or lower hypertension, proteinuria (protein $\leq 1+$ or $<2$ g of protein per 24 - $h$ urine collection), and grade 1 or lower bleeding, with no evidence of thrombosis or embolism. Treatment was discontinued in patients who had any of the following conditions: gastrointestinal perforation, grade 3 or higher hypertension, bleeding, thrombosis or embolism, hypersensitivity, and grade 4 or higher proteinuria. Treatment was delayed for up to 1 week if any of these requirements was not satisfied.

In patients who had hematologic toxicity (leukocyte count $<2,000 / \mu \mathrm{L}$, neutrophil count $<1,000 / \mu \mathrm{L}$, platelet count $<75,000 / \mu \mathrm{L}$, or serum total bilirubin level $\geq 2.5 \mathrm{mg} / \mathrm{dL}$ ) or grade 3 or higher severe non-hematologic toxicities, the doses of FU, S-1, and irinotecan were decreased by $20 \%$. If treatment could not be resumed within 28 days after withdrawal, treatment was discontinued.

\section{Assessments}

Pretreatment evaluation included a clinical history and physical examination, complete blood cell count, blood chemistry, urinalysis, electrocardiography, chest radiography, and CT of the chest and abdomen. During therapy, toxicity was assessed weekly according to the National Cancer Institute - Common Toxicity Criteria (NCI-CTC), version 4.0. Physical examination was conducted, and the results were recorded before each cycle, or more frequently if clinically indicated. Tumor response was assessed by 


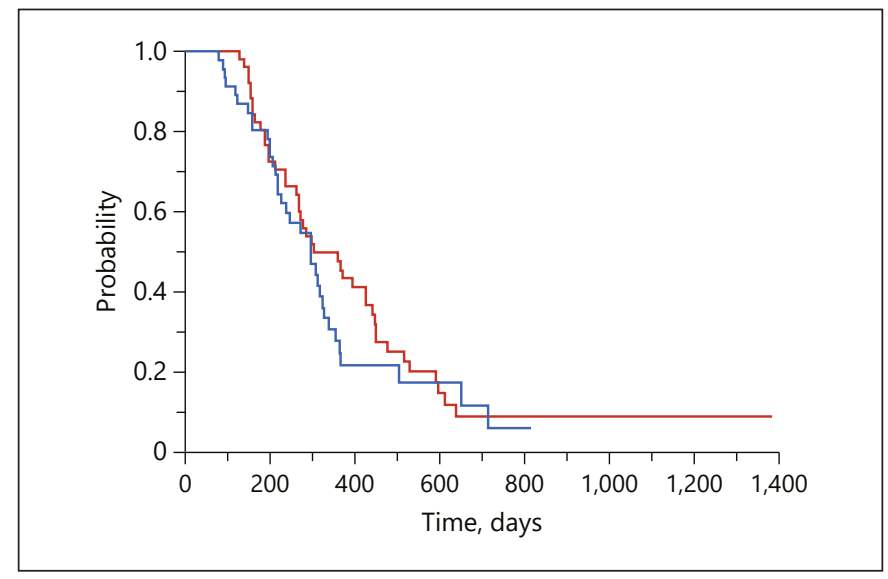

Fig. 1. Progression-free survival.

CT scans every 4 weeks. Efficacy was evaluated after every cycle according to the Response Evaluation Criteria in Solid Tumors (RECIST, version 1.1).

Statistical Analyses

The primary endpoint was the 1-year progression-free survival (PFS) rate. Secondary endpoints were PFS, overall response rate (ORR), overall survival (OS), and adverse events. The primary analysis was performed in the full analysis set. Safety analyses were performed in patients who had received the protocol treatment at least once.

Assuming that the lower threshold of the 1-year PFS rate was $30 \%$ in patients who had not previously received chemotherapy and that the expected 1 -year PFS rate was $70 \%$, with a one-side $a$ value of 0.05 and a $\beta$ value of 0.2 , we calculated the target number of previously untreated patients to be 46 in each groups. The target number of patients was therefore set at 100 , including dropouts and excluded patients.

Time-dependent events were analyzed by the Kaplan-Meier method. A log-rank test was used to compare the survival rates between the groups. Fisher's test was used to compare treatment responses between the groups.

This trial is registered with UMIN-CRT (http://www.umin. ac.jp/ctr/) (00001464).

\section{Results}

From December 2013 through January 2018, a total of 120 patients were randomly assigned to receive either a $24 \mathrm{~h}-\mathrm{SIRI} / \mathrm{B}$ regimen $(n=61)$ or to a FOLFIRI/B regimen $(n=59)$. Overall, 22 patients (10 patients in the $24 \mathrm{~h}-$ SIRI/B group and 12 patients in the FOLFIRI/B group) were not treated after randomization because of the patients' request $(n=7)$, ileus $(n=3)$, jaundice $(n=3)$, aggravation of general condition $(n=3)$, pneumonia $(n=$ 2 ), the presence of cancer in organs other than the col-

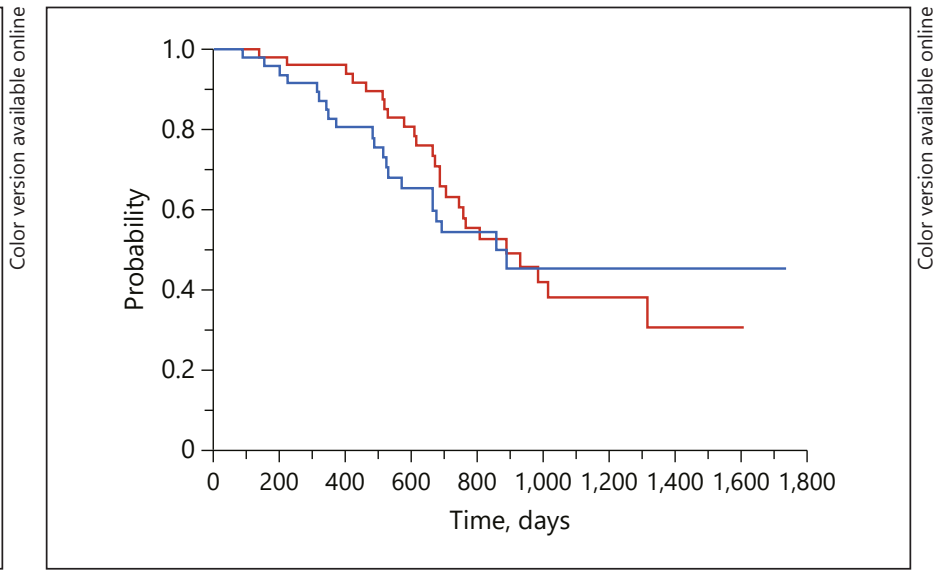

Fig. 2. Overall survival.

Table 1. Patient characteristics (full analysis set)

\begin{tabular}{lll}
\hline & $\begin{array}{l}24 \text { h-SIRI/B } \\
(n=51)\end{array}$ & $\begin{array}{l}\text { FOLFIRI/B } \\
(n=47)\end{array}$ \\
\hline Mean age (range), years & $65(23-79)$ & $64(38-83)$ \\
Performance status (0/1) & $37 / 14$ & $32 / 15$ \\
Sex (men/women) & $33 / 18$ & $28 / 19$ \\
Primary lesion (colon/rectum) & $28 / 23$ & $28 / 19$ \\
Metastatic organ (1/ 2 ) & $32 / 19$ & $24 / 23$ \\
Primary tumor (absent/present) & $35 / 16$ & $30 / 17$ \\
\hline
\end{tabular}

Values are given as $n$ unless indicated otherwise.

orectum $(n=2)$, perforation $(n=1)$, or heart failure $(n=$ 1).

The full analysis set population comprised 98 patients (51 patients in the $24 \mathrm{~h}-$ SIRI/B arm and 47 patients in the FOLFIRI/B arm). The cut-off date for primary analysis of the primary endpoint was November 2018. Patient characteristics were similar in both groups (Table 1). Two weeks of treatment was counted as one cycle in both groups. The median number of treatment cycles was 17 (range 4-58) in the 24h-SIRI/B group and 15 (range 2-44) in the FOLFIRI/B group.

The median follow-up period was 22.8 months (range 3.1-58.8). The 1-year PFS rate, the primary endpoint, was $43.14 \%$ in the $24 \mathrm{~h}-\mathrm{SIRI} / \mathrm{B}$ group and $19.15 \%$ in the FOLFIRI/B group (HR $=0.312$ [95\% CI 0.13-0.78], $p=$ 0.01; Fig. 1). The median PFS was 10.2 months (95\% CI 8.8-14.3) and 10.0 months (95\% CI 7.4-11.0), respectively $(p=0.375$; Fig. 1$)$. The median OS was 29.7 months (95\% CI 22.9-43.9), and 28.8 months (95\% CI 18.4 to ND), respectively ( $p=0.823$; Fig. 2 ). The ORR was 86.3 
Table 2. Overall response rate (RR)

\begin{tabular}{lccl}
\hline & $\begin{array}{l}24 \mathrm{~h}-\mathrm{SIRI} / \mathrm{B} \\
(n=51)\end{array}$ & $\begin{array}{l}\text { FOLFIRI/B } \\
(n=47)\end{array}$ & $\begin{array}{l}p \text { value } \\
\text { (Fisher's test) }\end{array}$ \\
\hline Complete response (CR) & 8 & 3 & \\
Partial response (PR) & 36 & 26 & \\
Stable disease (SD) & 0 & 2 & \\
Progressive disease (PD) & 7 & 16 & \\
RR & $86.3 \%$ & $61.7 \%$ & 0.0053 \\
DCR & $86.3 \%$ & $66.0 \%$ & 0.0177 \\
\hline
\end{tabular}
$\mathrm{SD})$.

RR: \% of CR + PR. DCR: Disease control rate (\% of CR + PR +

Table 3. Adverse events (all cycles)

\begin{tabular}{lrrrrr}
\hline & \multicolumn{2}{c}{24 h-SIRI/B $(n=51)$} & & \multicolumn{2}{c}{ FOLFIRI/B $(n=47)$} \\
\cline { 2 - 3 } \cline { 5 - 6 } \cline { 5 - 6 } & all & grade $\geq 3$ & & all & grade $\geq 3$ \\
\hline Leukopenia & 56.9 & 15.7 & & 55.3 & 12.8 \\
Neutropenia & 54.9 & 29.4 & & 53.2 & 23.4 \\
Hemoglobin & 72.5 & 3.9 & & 66.0 & 2.1 \\
Thrombocytopenia & 5.9 & 0.0 & & 4.3 & 0.0 \\
Liver & 19.6 & 0.0 & & 23.4 & 0.0 \\
Anorexia & 72.5 & 9.8 & & 72.3 & 2.1 \\
Nausea & 74.5 & 9.8 & 68.1 & 2.1 \\
Vomiting & 7.8 & 2.0 & 6.4 & 0.0 \\
Diarrhea & 60.8 & 11.8 & & 55.3 & 6.4 \\
Fatigue & 60.8 & 2.0 & 63.8 & 2.1 \\
Stomatitis & 43.1 & 0.0 & 27.7 & 0.0 \\
Alopecia & 17.6 & 0.0 & & 10.6 & 0.0 \\
Hypertension & 7.8 & 2.0 & 6.4 & 0.0 \\
\hline
\end{tabular}

and $61.7 \%$, respectively ( $p=0.0053$; Table 2$)$. Complete responses were obtained in 8 patients (15.7\%) and 3 patients $(6.4 \%)$, respectively.

Adverse events are summarized in Table 3. The incidence rates of grade 3 or higher hematologic toxicities were similar. The incidence rates of grade 3 or higher gastrointestinal toxicities, such as diarrhea, anorexia, and nausea, were higher in the $24 \mathrm{~h}-\mathrm{SIRI} / \mathrm{B}$ group.

\section{Discussion}

We hypothesized that a 24 -h biweekly intravenous infusion of irinotecan might be more effective and be associated with milder adverse events, based on characteristics of pharmacokinetics and pharmacodynamics of iri- notecan, and developed a $24 \mathrm{~h}-\mathrm{SIRI} / \mathrm{B}$ regimen. We adopted a 24-h biweekly intravenous infusion of irinotecan as a regimen for mCRC. We initially used oral UFT and subsequently used oral UFT plus leucovorin to perform clinical phase I and II studies. We also used oral S-1 and bevacizumab to conduct a clinical phase II study and reported that 24-h biweekly intravenous infusion of irinotecan combined with FUs (UFT or UFT plus leucovorin), S- 1 , and bevacizumab is useful with acceptable adverse events $[8,9,14]$.

The development of adverse events caused by irinotecan is closely related to metabolic enzyme activities. The expression of enzyme activity is largely controlled by gene levels. More than 60 types of uridine diphosphate glucuronosyltransferase (UGT)1A1 gene polymorphisms have been identified as key factors in the metabolism of irinotecan [15]. The most important gene polymorphism was considered UGT1A1*28. We previously reported on pharmacokinetics in patients who received irinotecan and S-1 and found that the recommended dose of irinotecan, given biweekly, was $125 \mathrm{mg} / \mathrm{m}^{2}$ in patients homozygous for the *28 allele [12] and that this regimen was highly active and well tolerated [13].

In the present study, we compared the effectiveness of a $24 \mathrm{~h}-\mathrm{SIRI} / \mathrm{B}$ regimen with that of a FOLFIRI/B regimen, a standard treatment, in previously untreated patients with advanced, recurrent CRC. In the $24 \mathrm{~h}-\mathrm{SIRI} / \mathrm{B}$ group, the 1-year PFS rate was $43.14 \%$. The median PFS and OS were 10.2 and 29.7 months, respectively. The treatment response rate was $86.3 \%$. In the FOLFIRI/B group, the 1 -year PFS rate was $19.15 \%$. The median PFS and OS were 10.0 and 28.6 months, respectively. The treatment response rate was $61.7 \%$. PFS did not differ largely between the groups. OS tended to be improved in the $24 \mathrm{~h}-\mathrm{SIRI} / \mathrm{B}$ group. The ORR was significantly higher in the $24 \mathrm{~h}$ SIRI/B group $(p=0.0053)$. Safety did not differ largely between the groups. The 24h-SIRI/B group had a higher incidence of gastrointestinal toxicities such as grade 3 or higher diarrhea, anorexia, and nausea.

Clinical phase II and III studies (FIRIS study) of patients who received secondary treatment for unresectable CRC demonstrated that combination treatment with oral S-1 and irinotecan (IRIS therapy) was not inferior to FOLFIRI therapy [16]. In patients with no history of treatment, combination treatment with IRIS and bevacizumab was associated with a high ORR and a longer PFS [17-19]. A recent controlled study (TRICOLORE trial) demonstrated that IRIS plus bevacizumab (a SIRI/B regimen) was equivalent to FOLFOX or CapeOx plus bevacizumab [20]. 
In the present study, we compared a 24-h SIRI/B regimen with a FOLFIRI/B regimen - a standard treatment - and found that the ORR was higher in the 24-h SIRI/B group. In the SIRI/B regimen in the TRICOLORE trial, irinotecan was given as a standard 90-min infusion, instead of a 24 -h continuous infusion. The ORR was $66.4 \%$ (control group, $70.6 \%$ ) [20]. In our study, the ORR was $86.3 \%$, suggesting that the use of a 24 -h continuous infusion of irinotecan was associated with an increase in the ORR.

The PFS did not differ largely between the groups, and the OS tended to be improved. The time to progressive disease from tumor shrinkage did not differ largely between the treatment groups. However, the complete RR was $15.7 \%$ in the $24 \mathrm{~h}$-SIRI/B group and $6.4 \%$ in the FOLFIRI/B group. The partial RR was $70.6 \%$ in the $24 \mathrm{~h}-$ SIRI/B group and $55.3 \%$ in the FOLFIRI/B group. These results suggested that the survival period may be longer in the $24 \mathrm{~h}-\mathrm{SIRI} / \mathrm{B}$ group. However, details remain unknown.

Safety did not differ largely between the groups. However, the $24 \mathrm{~h}-\mathrm{SIRI} / \mathrm{B}$ group had higher incidences of gastrointestinal toxicities such as grade 3 or higher diarrhea, anorexia, and nausea. One possible cause is that the combined drugs differed between the groups ( $\mathrm{S}-1$ vs. $5-\mathrm{FU}$ plus leucovorin). In the SIRI/B regimen in the TRICOLORE trial, the incidences of adverse events were as follows: $62.3 \%$ for any grade of diarrhea, $13.4 \%$ for grade 3 or higher diarrhea, $59.8 \%$ for any grade of anorexia, $6.7 \%$ for grade 3 or higher anorexia, $56.9 \%$ for any grade of nausea, and 3.3\% for grade 3 or higher nausea [20]. In our study, the incidences of adverse events associated with the $24 \mathrm{~h}-\mathrm{SIRI} / \mathrm{B}$ regimen were as follows: $60.8 \%$ for any grade of diarrhea, $11.8 \%$ for grade 3 or higher diarrhea, $72.5 \%$ for any grade of anorexia, 9.8\% for grade 3 or higher anorexia, $74.5 \%$ for any grade of nausea, and $9.8 \%$ for grade 3 or higher nausea. In other words, the use of 24 -h continuous infusion of irinotecan did not lower the incidences of gastrointestinal toxicities, suggesting that S-1 combined with 24-h continuous infusion of irinotecan may not lower the incidence of adverse events.

At present, chemotherapeutic strategies for advanced or recurrent CRC are determined according to the patients' condition. In particular, our regimen was associated with a high RR. Therefore, we believe that our regimen can be used as a treatment option in patients with advanced disease who started treatment to convert to surgical resection.

The limitations of our study are as follows: our study is a randomized controlled phase II trial but not a confir- matory trial; treatment outcomes were obtained from a single study, and patients in whom metastasis or recurrence developed only in the liver were not enrolled.

In our study, a $24 \mathrm{~h}-\mathrm{SIRI} / \mathrm{B}$ regimen, given as a firstline chemotherapy for $\mathrm{mCRC}$, was equivalent to a FOLFIRI/B regimen with regard to the incidences of adverse events. However, the $24 \mathrm{~h}-\mathrm{SIRI} / \mathrm{B}$ regimen was associated with a significantly higher RR and improved 1-year PFS.

\section{Acknowledgement}

Nothing to be declared.

\section{Statement of Ethics}

This trial was approved by the Institutional Review Board of Tokai University School of Medicine (13R-155). Our protocol was designed according to the Declaration of Helsinki and ethical guidelines. All patients provided written informed consent before enrollment.

\section{Disclosure Statement}

All authors have no potential conflicts of interest to declare.

\section{Funding Sources}

There was no funding source for this study.

\section{Author Contributions}

Conception and design: S. Sadahiro and T. Suzuki. Provision of study materials or patients: all authors. Collection and assembly of data: T. Suzuki, K. Okada, and S. Sadahiro. Data analysis and interpretation: S. Sadahiro and K. Okada. Manuscript writing: S. Sadahiro and T. Suzuki. Final approval of manuscript: all authors.

References

1 Van Cutsem E, Cervantes A, Nordlinger B, Arnold D. Metastatic colorectal cancer: ESMO Clinical Practice Guidelines for diagnosis, treatment and follow-up. Ann Oncol. 2014 Sep;25(Suppl 3:iii1-9.

2 National Comprehensive Cancer Network (NCCN). Colon Cancer [Internet]. [cited 2018 Oct 19]. Available from: https://www. nccn.org/professionals/physician_gls/pdf/ colon.pdf. 
3 Watanabe T, Muro K, Ajioka Y, Hashiguchi Y, Ito Y, Saito Y, et al. Japanese Society for Cancer of the Colon and Rectum (JSCCR) guidelines 2016 for the treatment of colorectal cancer. Int J Clin Oncol. 2020 Jan;25(1):1-42.

4 Yamazaki K, Nagase M, Tamagawa H, Ueda S, Tamura T, Murata K, et al. Randomized phase III study of bevacizumab plus FOLFIRI and bevacizumab plus mFOLFOX6 as firstline treatment for patients with metastatic colorectal cancer (WJOG4407G). Ann Oncol. 2016 Aug;27(8):1539-46.

5 André T, Boni C, Navarro M, Tabernero J, Hickish T, Topham C, et al. Improved overall survival with oxaliplatin, fluorouracil, and leucovorin as adjuvant treatment in stage II or III colon cancer in the MOSAIC trial. J Clin Oncol. 2009 Jul 1;27(19):3109-16.

6 Houghton PJ, Cheshire PJ, Hallman JD 2nd, Lutz L, Friedman HS, Danks MK, et al. Efficacy of topoisomerase I inhibitors, topotecan and irinotecan, administered at low dose levels in protracted schedules to mice bearing xenografts of human tumors. Cancer Chemother Pharmacol. 1995;36(5):393-403.

7 Rothenberg ML, Kuhn JG, Schaaf LJ, Drengler RL, Eckhardt SG, Villalona-Calero MA, et al. Alternative dosing schedules for irinotecan. Oncology (Williston Park, NY). 1998 Aug;12(8 Suppl 6):68-71.

8 Sadahiro S, Suzuki T, Maeda Y, Ishikawa K, Yasuda S, Makuuchi H, et al. Phase I/II study of 24-hour infusion of irinotecan combined with oral UFT for metastatic colorectal cancer. Chemotherapy. 2008;54(2):140-6.
9 Sadahiro S, Suzuki T, Maeda Y, Tanaka A, Ishikawa $\mathrm{K}$, Makuuchi $\mathrm{H}$, et al. Phase I/II study of twenty-four-hour infusion of irinotecan in combination with oral UFT plus leucovorin for metastatic colorectal cancer. Oncology. 2009;76(5):338-41.

10 Schöffski P. The modulated oral fluoropyrimidine prodrug S-1, and its use in gastrointestinal cancer and other solid tumors. Anticancer Drugs. 2004 Feb;15(2):85-106.

11 Shirasaka T, Shimamato Y, Ohshimo H, Yamaguchi M, Kato T, Yonekura K, et al. Development of a novel form of an oral 5-fluorouracil derivative ( $\mathrm{S}-1)$ directed to the potentiation of the tumor selective cytotoxicity of 5 -fluorouracil by two biochemical modulators. Anticancer Drugs. 1996 Jul;7(5):548-57.

12 Sadahiro S, Maeda Y, Suzuki T, Tanaka A, Ogoshi K, Kamijo A, et al. Phase I/pharmacokinetic (PK) study of biweekly 24-hour infusion of irinotecan (irinotecan) plus S-1 for metastatic colorectal cancer (MCRC): role of UGT1A1 polymorphisms. Poster presented at: 2010 ASCO Gastrointestinal Cancers Symposium. 2010 Jan 22-24; Orlando, USA; Poster: 398

13 Sadahiro S, Suzuki T, Tanaka A, Okada K, Saito G, Kamijo A. A phase II trial of combined chemotherapy with oral S-1 and 24hour infusions of irinotecan plus bevacizumab in patients with metastatic colorectal cancer. Oncology. 2015;88(6):353-9.

14 Sadahiro S, Suzuki T, Tanaka A, Okada K, Saito G, Kamijo A, et al. Phase II study of preoperative concurrent chemoradiotherapy with S-1 plus bevacizumab for locally advanced resectable rectal adenocarcinoma. Oncology. 2015;88(1):49-56.
15 Ando Y, Hasegawa Y. Clinical pharmacogenetics of irinotecan (CPT-11). Drug Metab Rev. 2005;37(3):565-74.

16 Muro K, Boku N, Shimada Y, Tsuji A, Sameshima S, Baba $\mathrm{H}$, et al. Irinotecan plus S-1 (IRIS) versus fluorouracil and folinic acid plus irinotecan (FOLFIRI) as second-line chemotherapy for metastatic colorectal cancer: a randomised phase $2 / 3$ non-inferiority study (FIRIS study). Lancet Oncol. 2010 Sep; 11(9):853-60.

17 Komatsu Y, Yuki S, Sogabe S, Fukushima H, Nakatsumi H, Kobayashi Y, et al. Phase II study of combined chemotherapy with irinotecan and S-1 (IRIS) plus bevacizumab in patients with inoperable recurrent or advanced colorectal cancer. Acta Oncol. 2012 Sep; 51(7):867-72.

18 Yamada $\mathrm{Y}$, Yamaguchi T, Matsumoto $\mathrm{H}$ Ichikawa Y, Goto A, Kato K, et al. Phase II study of oral S-1 with irinotecan and bevacizumab (SIRB) as first-line therapy for patients with metastatic colorectal cancer. Invest $\mathrm{New}$ Drugs. 2012 Aug;30(4):1690-6.

19 Mizushima T, Ide Y, Murata K, Ohashi I, Yasumasa K, Fukunaga M, et al. A phase II study of combined chemotherapy with 5-week cycles of S-1 and CPT-11 plus bevacizumab in patients with metastatic colon cancer. Oncology. 2013;85(6):317-22.

20 Yamada Y, Denda T, Gamoh M, Iwanaga I, Yuki S, Shimodaira $\mathrm{H}$, et al. S- 1 and irinotecan plus bevacizumab versus mFOLFOX6 or CapeOX plus bevacizumab as first-line treatment in patients with metastatic colorectal cancer (TRICOLORE): a randomized, openlabel, phase III, noninferiority trial. Ann Oncol. 2018 Mar 1;29(3):624-31. 